\title{
Numerical analysis of the shielding effect provided by periodic elastic scatterers
}

\author{
Paulo Amado-Mendes ${ }^{1, *}$, Luís Godinho ${ }^{1}$, Pedro Alves-Costa ${ }^{2}$, Alexandre Castanheira- \\ Pinto $^{2}$, and Carlos Albino ${ }^{1}$ \\ ${ }^{1}$ ISISE, Department of Civil Engineering, University of Coimbra, Portugal \\ ${ }^{2}$ CONSTRUCT., Faculty of Engineering of the University of Porto, Portugal
}

\begin{abstract}
The search for innovative mitigation solutions that can be adopted in urban areas to efficiently reduce the effects of induced vibrations by increasing numbers of vehicles has motivated a significant number of researchers from different fields. An interesting concept seems to be the use of buried elastic inclusions, periodically arranged parallel to the ground surface, applying sonic crystal inspired technologies and bandgap filtering on adequate frequency ranges. In previous works, preliminary numerical results have shown the influence of the presence of multiple elastic heterogeneities on the attenuation of the vibration fields in receivers located on the opposite side from the traffic vibration source. Following this concept, a 2.5D numerical model has been implemented, based on the Finite Element Method (FEM) and on the use of a numerical absorbing layer. The effects on the vibration levels provided by a set of periodic elastic inclusions inserted in the host propagation medium are parametrically analysed. In fact, frequency domain results, obtained when modelling different configurations of the periodic structure and host soil, are used to assess the efficiency of these arrays as a mitigation measure.
\end{abstract}

\section{Introduction}

Vibration mitigation and protection is an important problem that is related to human comfort and that has been accepted as a relevant worldwide societal challenge. Novel protection techniques have been investigated, namely a new generation of mitigation measures that include the so-called metamaterials [1]. These resources are, in fact, artificial materials designed, fabricated or arranged in such a way that they exhibit behaviours not found in nature. One practical application for this type of systems is acoustic attenuation, as proposed by Umnova et al and Sánchez Dehesa et al [2,3]. The good results that have been registered in noise control applications make it possible to believe that similar concepts could successfully be adopted for vibration mitigation in elastic soils, making use of buried metamaterial-like structures.

Some recent research works can be found in the literature, regarding seismic protection and vibration mitigation by periodic structures. Alagoz and Alagoz [4] presented numerical simulations to show the shielding effect of what they called "seismic crystals", indicating

\footnotetext{
*Corresponding author: pamendes@dec.uc.pt
} 
an effective attenuation of seismic waves and consequent damage reduction in case of an earthquake. On the other hand, Kim and Das [5] developed a meta-barrier composed of many periodic meta-boxes to reduce the amplitude of seismic waves and create an area of protection tested by computer simulation. Brûlé et al $[6,7]$ reported what is possibly the first evidence of the efficiency of periodic buried structures as a shield to seismic waves, after performing a controlled seismic test on a soil with a grid of bored vertical, cylindrical and empty inclusions. Krodel et al [8] combined the numerical analysis and scaled experiments in the study of an array of resonating structures buried around large buildings to control the propagation of seismic waves. Preliminary works $([9,10])$ by the authors of the present paper have also demonstrated the vibration mitigation properties of arrays of elastic scatterers, in a reduced number of scenarios. Consequently, this concept for vibration protection based on buried metamaterials may constitute a powerful promising future solution that deserves attention.

In this work, a brief description of the $2.5 \mathrm{D}$ implemented numerical model is initially given. Then, the numerical example is presented and the solutions for the reference cases are illustrated. A parametric study is shortly presented in order to analyse the main features of the physical system incorporating periodic elastic inclusions and preliminarily verify its mitigation effects under different configurations. The paper ends with some final remarks.

\section{Numerical formulation -2.5D FEM model with absorbing layers}

Consider an elastic medium where a set of inclusions are introduced, representing a multidomain elastic system. The wave equation in the space-frequency domain can be written as

$$
(\lambda+2 \mu) \nabla \nabla \cdot \mathbf{u}-\mu \nabla \times \nabla \times \mathbf{u}+\omega^{2} \rho \mathbf{u}=0
$$

where $\mathbf{u}$ represents the displacement vector, $\lambda$ and $\mu$ correspond to the Lamé's constants of the elastic medium and $\rho$ the mass density, and $\omega$ the exciting angular frequency.

When modelling many civil engineering problems, like transportation infrastructures, the physical system and the solid inclusions can be assumed as infinite and with a geometry that does not change along the $z$ longitudinal direction. If this physical system is excited by a harmonic dilatational point source with an angular frequency $\omega$, a $2.5 \mathrm{D}$ numerical approach can be adopted, after applying a Fourier transformation along the longitudinal direction. Hence, the 3D displacement field can be evaluated as a continuous integral of simpler 2D solutions in the frequency domain by:

$$
\mathbf{u}^{3 D}=\int_{-\infty}^{+\infty} \mathbf{u}^{2.5 D}\left(k_{z}\right) \mathrm{e}^{-\mathrm{i} k_{z}\left(z-z_{0}\right)} \mathrm{d} k
$$

with $k_{z}$ representing the longitudinal wavenumber along the $z$ direction. This integral can be approximated by a discrete summation (assuming an infinite set of virtual sources equally spaced in the $z$ axis) that converges within a finite number of terms [11].

The solution of the 2.5D problem is obtained in this work by the Finite Element Method (FEM), with the different elastic domains being discretised, namely, the host medium (or media) and the elastic inclusions periodically organized and embedded in the host soil(s). In this 2.5D FEM approach, the modelling domain is discretised along the cross-section of the physical problem while the solution in the remaining (longitudinal) direction is determined by the above referred Fourier transformation, with the numerical problem being solved in the wavenumber-frequency domain. 
Following the classic finite elements approach, the equilibrium of the domain can be established by the following equation, given in terms of nodal variables $[12,13]$ :

$$
\left(\mathbf{K}-\omega^{2} \mathbf{M}\right) \mathbf{u}\left(k_{z}, \omega\right)=\mathbf{F}\left(k_{z}, \omega\right)
$$

where $\mathbf{K}$ and $\mathbf{M}$ represent the stiffness and mass matrices, and vectors $\mathbf{u}$ and $\mathbf{F}$ correspond to the nodal displacements and to the external nodal forces in the transformed domain, given in [13]. In order to improve the computational efficiency, the stiffness matrix $\mathbf{K}$ can be divided into three sub-matrices, independent of the wavenumber and frequency, and equation (3) can then be substituted by the global equation to be solved:

$$
\left(\mathbf{K}_{1}+\mathrm{i} k_{z} \mathbf{K}_{2}+k_{z}^{2} \mathbf{K}_{3}-\omega^{2} \mathbf{M}\right) \mathbf{u}\left(k_{z}, \omega\right)=\mathbf{F}\left(k_{z}, \omega\right)
$$

Analyses of the 3D results, both in the frequency domain and in the time domain, can be performed after the application of one or two inverse Fourier transformations to the obtained FEM results. This procedure can be very efficient in comparison to a classical 3D FEM approach and also reveals to be very useful in the interpretation of different observed phenomena in longitudinally invariant physical systems.

Additionally, in the present work, an alternative method suggested by Semblat et al [14] has been implemented in order to efficiently avoid spurious wave reflections in the artificial mesh boundaries. Therefore, numerical absorbing layers (with spatially variable damping coefficients) have been defined in the outer part of the elastic domain being modelled where the unbounded soil is truncated. This simple procedure has demonstrated to be easy to implement and very efficient for elastic waves with different incidence directions.

The 2.5D FEM model has been successfully verified by comparing with analytical solutions and other numerical methods previously proposed by the authors [11, 15], but in the context of the present text, for the sake of brevity, these results are not presented.

\section{Parametric studies}

The proposed 2.5D FEM model is a useful numerical tool that is now applied to a set of parametric analyses of the wave propagation patterns and physical behaviour of the modelled systems, composed by different geometric configurations and numbers of buried inclusions. A very limited selection of the extensive sets of results is here presented for distinct geometries, arrangements of circular cylindrical inclusions and materials characterizing the diverse elastic media. In Table 1, the material properties used in this work are summarized, as well as the correspondent wave propagation velocities. The results are analysed for a range of frequencies (from 10 to $100 \mathrm{~Hz}$ ) and a range of phase velocity values (from 100 to $500 \mathrm{~m} / \mathrm{s}$ ), defined by $c=\omega / k_{z}$, and the amplitude values represented in the illustrated figures are determined by the displacement norm, combining the 3 orthogonal directions $(x, y$ and $z)$ and numerically evaluated by $|u|=\sqrt{u_{x}^{2}+u_{y}{ }^{2}+u_{z}^{2}}$.

Table 1. Material properties adopted in the studies and wave propagation velocities.

\begin{tabular}{|c|c|c|c|c|c|c|}
\hline Material & $\begin{array}{c}\text { Young } \\
\text { modulus } \\
E(\mathrm{GPa})\end{array}$ & $\begin{array}{c}\text { Poisson } \\
\text { coeff. }\end{array}$ & $\begin{array}{c}\text { Density } \\
\rho\left(\mathrm{kg} / \mathrm{m}^{3}\right)\end{array}$ & $\begin{array}{c}\mathrm{P} \text { wave } \\
c_{p}(\mathrm{~m} / \mathrm{s})\end{array}$ & $\begin{array}{c}\mathrm{S} \text { wave } \\
c_{S}(\mathrm{~m} / \mathrm{s})\end{array}$ & $\begin{array}{c}\text { Rayleigh } \\
\text { wave } \\
c_{R}(\mathrm{~m} / \mathrm{s})\end{array}$ \\
\hline Host soil $\left(\Omega_{1}\right)$ & 116 & 0.33 & 1700 & 318.0 & 160.2 & 149.1 \\
\hline Ballast $\left(\Omega_{3}\right)$ & 97 & 0.12 & 1591 & 251.1 & 165.0 & 147.1 \\
\hline
\end{tabular}




\begin{tabular}{|c|c|c|c|c|c|c|}
\hline Sub-ballast $\left(\Omega_{3}\right)$ & 212 & 0.30 & 1913 & 386.2 & 206.5 & 191.2 \\
\hline $\begin{array}{c}\text { Hard Inclusions } \\
- \text { Concrete }\left(\Omega_{2}\right)\end{array}$ & 30 & 0.30 & 2500 & 4019.2 & 2148.3 & 1989.7 \\
\hline
\end{tabular}

\subsection{Examples definition and reference solutions}

In the examples used for the parametric studies, a symmetric model is considered along the cross-sectional plane, with symmetry in respect to a vertical axis crossing the origin of the $(x, y)$ axes, which allows the efficient application of the FEM formulation previously described (for a schematic representation, see Figure 1). A set of two rows with four solid inclusions $(4 \times 2)$ is buried in an elastic half-space soil (referred in Table 1 and Figure 1 as material/domain $\Omega_{1}$ ), which is excited by a harmonic load applied along the vertical direction on the surface of the host soil, at point $\left(x_{0}=0 \mathrm{~m}, y_{0}=0 \mathrm{~m}\right)$. The elastic inclusions are assumed to be circular (with radius equal to $0.3 \mathrm{~m}$ ) and infinitely long in the $z$ direction, being buried at a depth of $0.2 \mathrm{~m}$ from the surface and completely filled with a homogeneous material (material/domain $\Omega_{2}$ ). Initially, hard inclusions made of concrete were considered, with the correspondent centres equally spaced of $1 \mathrm{~m}$ along both $x$ and $y$ directions, and the closest inclusion to the source centred at $x_{c}=-5 \mathrm{~m}$. The inclusions can be directly buried in the host soil or can eventually be embedded in a confined soil near the set of inclusions, with properties of ballast or sub-ballast materials (material/domain $\Omega_{3}$ ).

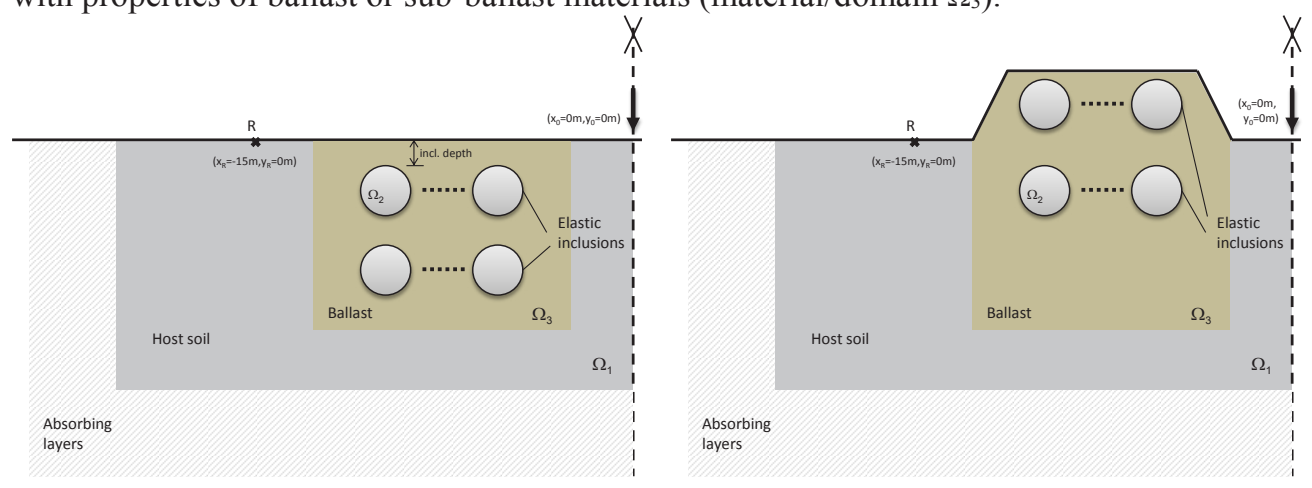

Fig. 1. Problem representation and general scheme definition on a cross-sectional $x$-y plane view: (left) half-space system without embankment; (right) half-space system with embankment.

Figure 2 presents the amplitude of the displacement norm computed at receiver $\mathrm{R}$, located at $\left(x_{R}=-15 \mathrm{~m}, y_{R}=0 \mathrm{~m}\right)$, for the reference cases where no elastic inclusions are introduced and therefore the wave propagation occurs in two homogeneous media: the elastic half-space with flat surface (without embankment) and the elastic half-space with an embankment, that elevates the free surface in a limited region. In the first case (left side of Figure 2), it is clear that, for phase velocity values below the propagation velocity of the Rayleigh wave $\left(c_{R}\right)$, very little energy propagates in the system (null displacements observed for all frequency range), which only enables evanescent waves. For phase velocity values above $c_{R}$, the elastic waves propagate without obstacles and higher displacements values are observed. In the second case, e.g. in the presence of the embankment, the registered results change significantly for phase velocity values above $c_{R}$, as can be seen on the right side of Figure 2. Here, the displacement amplitude values decrease for frequencies above around $40 \mathrm{~Hz}$, as a consequence of the presence of the embankment and the resulting discontinuity of the half-space surface. 


\subsection{Set of periodic inclusions without and with embankment}

To verify the effect of the presence of the set of $4 \times 2$ hard inclusions buried in the host soil, Figure 3 presents the amplitude of the displacement norm determined at the same receiver $\mathrm{R}$, selected so as to place the set of elastic inclusions between the source and the receiver. By comparing Figures 2 and 3, one can assess the protection effect introduced by the periodic inclusions, more evident without the presence of embankment. The presence of solid obstacles significantly reduces the amplitude of the displacements in a region of the $c-f$ domain of analysis influenced by the vibration modes of the inclusions (this is confirmed by the dispersion curve for longitudinal bending waves also plotted as dashed black lines for a concrete inclusion). However, in these plots, it is not easy to quantify the effect related to the Bragg effect due to the periodic distribution of the inclusions. Some effects of the assumed cross-section symmetry can be observed as lighter lines below the dispersion curves and approximating the region with phase velocity values near $c_{R}$.
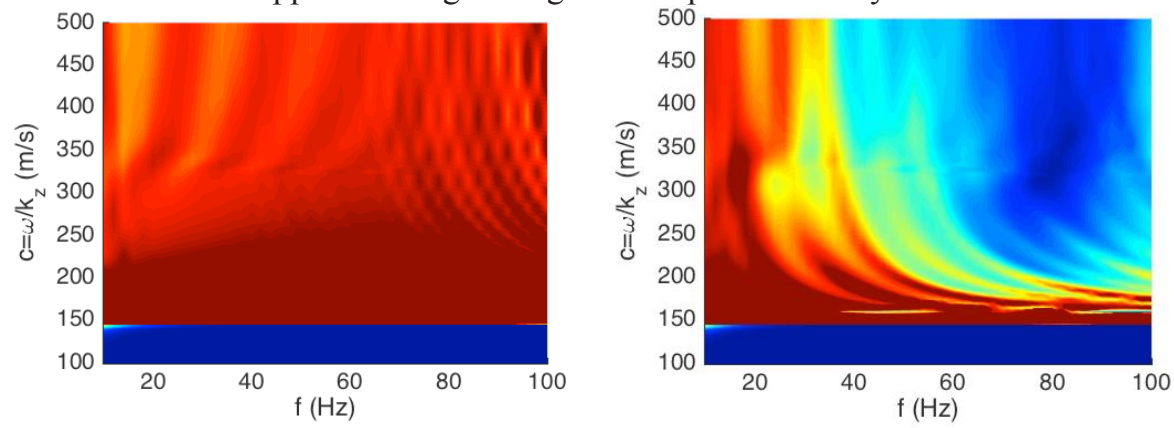

Fig. 2. Representation of displacement norm at receiver $\mathrm{R}$ for the reference cases without periodic inclusions - homogeneous media: (left) without embankment; (right) with embankment.
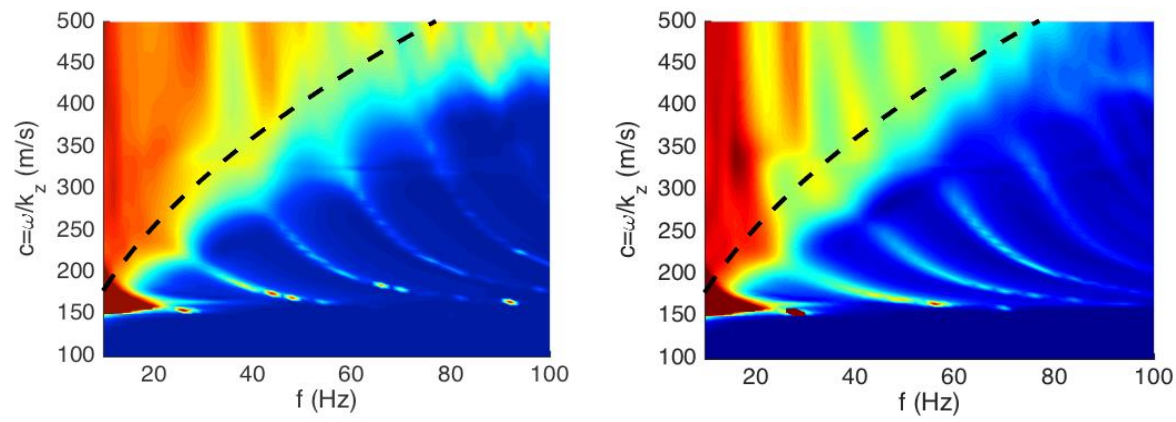

Fig. 3. Representation of displacement norm at receiver $\mathrm{R}$ for the cases with two rows of periodic inclusions embedded in host soil: (left) without embankment; (right) with embankment.

\subsection{Set of periodic inclusions with different stiffness}

As expected, the stiffness of the inclusions has a great influence in the dynamic response of the systems being analysed. This can be verified in Figure 4, where the amplitude of the displacement norm is plotted for the cases with the inclusions being modelled with a softer material, characterized by a reduced Young modulus by a factor of eight. The dispersion relation for each inclusion has also been recalculated and plotted as dashed black lines, exhibiting a very different behaviour, with reduced attenuation efficiency, but once again clearly delimiting (to lower phase velocity values) the region of the $c-f$ domain where the protection effect by the group of inclusions is more evident. 

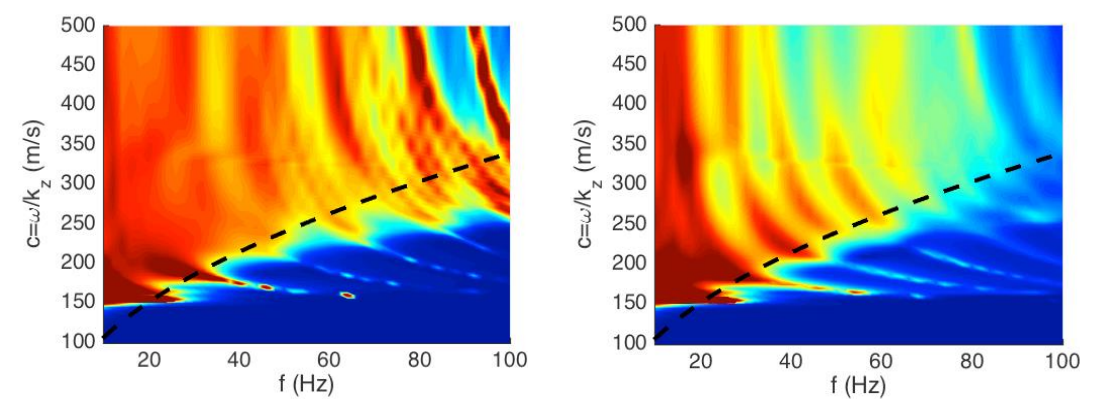

Fig. 4. Representation of displacement norm at receiver $\mathrm{R}$ for the cases with two rows of periodic soft inclusions embedded in host soil: (left) without embankment; (right) with embankment.

\section{Final considerations}

The present paper describes a preliminary parametric study, based on a 2.5D FEM model, with the objective of understanding the shielding effect provided by a set of periodic circular cylindrical inclusions buried near the surface of a host soil. The results obtained show the influence of a selected number of parameters modifying the mitigation effect introduced by the buried periodic inclusions.

The authors acknowledge the financial support of FCT - Foundation for Science and Technology and COMPETE, through research project PTDC/ECM-COM/1364/2014 (META_Shield). This work is also financed by FEDER funds through the Competitivity Factors Operational Programme COMPETE and by national funds through FCT within the scope of the projects POCI-01-0145FEDER-007633 and POCI-01-0145-FEDER-007457, and through the Regional Operational Programme CENTRO2020 within the scope of the project CENTRO-01-0145-FEDER-000006.

\section{References}

[1] P.A. Deymier (ed.), Acoustic Metamaterials and Phononic Crystals (Springer, 2013)

[2] O. Umnova, K. Attenborough, C. M. Linton, J. Acoust. Soc. Am. 119, 1 (2006)

[3] J. Sánchez-Dehesa, V.M. Gacia-Chocano, D. Torrent, F. Cervera, S. Cabrera, F. Simon, J. Acoust. Soc. Am. 129 (2011)

[4] B.B. Alagoz, S. Alagoz, S., Open Journal of Acoustics 1 (2011)

[5] S.-H. Kim, M.P. Das, Mod. Phys. Lett. B 27 (2013)

[6] S Brûlé, E Javelaud, S Enoch, S Guenneau, arXiv preprint arXiv:1301.7642 (2013)

[7] S. Brûlé, E.H. Javelaud, S. Enoch, S. Guenneau, Phys. Rev. Lett. 112 (2014)

[8] S. Kroedel, N. Thome, C. Daraio, Extreme Mechanics Letters 4 (2015)

[9] P. Amado-Mendes, L. Godinho, in 11th Int. Conf. on Vibration Problems (ICOVP), Lisbon (2013)

[10] C. Albino, L. Godinho, D. Dias-da-Costa, P, Amado Mendes, in 22nd Int. Cong. on Acoustics (ICA2016), Buenos Aires (2016)

[11] P. Amado-Mendes, P. Alves Costa, L. Godinho, P. Lopes, Eng. Struct. 104 (2015)

[12] Y.B. Yang, H.H. Hung, Int. J. Numer. Methods Eng. 51 (2001)

[13] P. Alves Costa, R. Calçada, A. Silva Cardoso, A. Bodare, Soil Dyn. Earthq. Eng. 30, 4 (2010)

[14] J.-F. Semblat, A. Gandomzadeh, L. Lenti, C.R. Mecanique 338, 1 (2010)

[15]P. Lopes, P. Alves-Costa, R. Calçada, A. Silva Cardoso, in Traffic Induced Environmental Vibrations and Controls: Theory and Application, (2013) 